\title{
AGN Feedback Driven Molecular Outflow in NGC 1266
}

K. Alatalo ${ }^{1}$, K. E. Nyland ${ }^{2}$, G. Graves ${ }^{1}$, S. Deustua ${ }^{3}$, J. Wrobel, ${ }^{4}$ L. M. Young ${ }^{2}$, T. A. Davis ${ }^{5}$, M. Bureau ${ }^{6}$, E. Bayet,${ }^{6}$ L. Blitz, ${ }^{2}$ M. Bois,${ }^{7}$ F. Bournaud, ${ }^{8}$, M. Cappellari,${ }^{6}$ R. L. Davies,${ }^{6}$ P. T. de Zeeuw ${ }^{5,14}$ E. Emsellem, ${ }^{5,17}$ S. Khochfar, ${ }^{9}$ D. Krajnovic ${ }^{5}$ H. Kuntschner, ${ }^{5}$ S. Martín, ${ }^{10}$ R. M. McDermid, ${ }^{11}$ R. Morganti, ${ }^{12}$ T. Naab, ${ }^{9}$ M. Sarzi, ${ }^{13}$ N. Scott, ${ }^{15}$ P. Serra ${ }^{13}$ and A. Weijmans ${ }^{16}$

${ }^{1}$ University of California, Berkeley, USA; ${ }^{2}$ New Mexico Tech, Socorro, USA; ${ }^{3}$ Space Telescope Science Institute, Baltimore, USA; ${ }^{4} \mathrm{NRAO}$, Socorro, USA; ${ }^{5}$ ESO, Garching, Germany; ${ }^{6}$ University of Oxford, UK; ${ }^{7}$ Observatoire de Paris, France; ${ }^{8}$ Université Paris Diderot, France;

${ }^{9}$ MPI for Extraterrestrial Physics, Garching, Germany; ${ }^{10}$ ESO, Santiago, Chile; ${ }^{11}$ Gemini Observatory, Hilo, USA; ${ }^{12}$ ASTRON, Dwingeloo, The Netherlands; ${ }^{13}$ University of

Hertfordshire, Hatfield, UK; ${ }^{14}$ Leiden University, The Netherlands; ${ }^{15}$ Swinburne University, Australia; ${ }^{16}$ University of Toronto, Canada; ${ }^{17}$ Université de Lyon, France

\begin{abstract}
NGC 1266 is a nearby field galaxy observed as part of the ATLAS ${ }^{3 \mathrm{D}}$ survey (Cappellari et al. 2011). NGC 1266 has been shown to host a compact ( $<200$ pc) molecular disk and a mass-loaded molecular outflow driven by the AGN (Alatalo et al. 2011). Very Long Basline Array (VLBA) observations at $1.65 \mathrm{GHz}$ revealed a compact (diameter $<1.2 \mathrm{pc}$ ), high brightness temperature continuum source most consistent with a low-level AGN origin. The VLBA continuum source is positioned at the center of the molecular disk and may be responsible for the expulsion of molecular gas in NGC 1266. Thus, the candidate AGN-driven molecular outflow in NGC 1266 supports the picture in which AGNs do play a significant role in the quenching of star formation and ultimately the evolution of the red sequence of galaxies.
\end{abstract}

Keywords. Galaxies - Active Galactic Nuclei - ISM

\section{Introduction}

The present-day galaxy population has a bimodal color distribution, with a genuine lack of green valley galaxies (Strateva et al. 2001; Baldry et al. 2004). This dearth of green valley galaxies, coupled with the increase in quiescent galaxies since $z \sim 1$, implies that galaxies quench star formation (SF) and transition rapidly from blue to red optical colors (Bell et al. 2004; Faber et al. 2007). Thus far, it is not clear if, or how, AGN feedback plays a role in this rapid quenching (Springel, di Matteo \& Hernquist 2005; Hopkins et al. 2005), though there is some circumstantial evidence that supports the AGN-driven SF quenching scenario. In particular, Schawinski et al. (2007) have found that AGNs predominantly reside in green valley galaxies, but direct evidence (e.g., a galaxy where the AGN is clearly responsible for the expulsion of starforming material) has been scarce. However, the recent discovery of a massive molecular outflow likely driven by an AGN in the early-type galaxy NGC 1266 may be a rare example of a galaxy transitioning from the blue to red sequence. NGC 1266 is thus a promising local laboratory for studying AGN-driven SF quenching.

\section{Overview}

NGC 1266 is a nearby $(D \approx 29.9 \mathrm{Mpc})$ field early-type galaxy that CO observations have shown to harbor a compact molecular disk $\left(\Sigma_{\text {gas }}>10^{4} M_{\odot} \mathrm{pc}^{-2}\right)$ and a 
mass-loaded $\left(M_{\text {gas }}>10^{9} M_{\odot} ; \dot{M}>13 M_{\odot} \mathrm{yr}^{-1}\right)$ molecular outflow (Alatalo et al. 2011). Alatalo et al. (2011) showed that the molecular gas could not be driven by SF alone and concluded that an AGN was the most likely culprit. Very Large Array (VLA) observations revealed a compact radio core coincident with the weak detection of hard X-rays with Chandra (Alatalo et al. 2011). Subsequent VLBA observations confirmed the presence of a compact, high brightness temperature $\left(T_{b}>10^{7} \mathrm{~K}\right)$ radio core (Fig. 1), further supporting existing evidence that NGC 1266 hosts a low-level AGN. The VLBA source is positioned at the center of the molecular disk (Fig. 1a, yellow contours), where the $\mathrm{H}_{2}$ column density is at least $6 \times 10^{24} \mathrm{~cm}^{-2}$. Given the high column density of molcular gas directly atop the AGN, it is unsurprising that little hard X-ray emission was detected (Alatalo et al. 2011) since this object may be mildly compton thick. VLBA observations have provided strong supporting evidence for the presence of an AGN in the center of NGC 1266, evidence directly implicating the AGN in the outflow awaits . Future High Sensitivity Array observations will provide improved imaging capable of revealing the signature of a radio outflow at the launch point of the outflowing molecular gas and would provide direct evidence of an AGN-driven molecular outflow in the local universe.

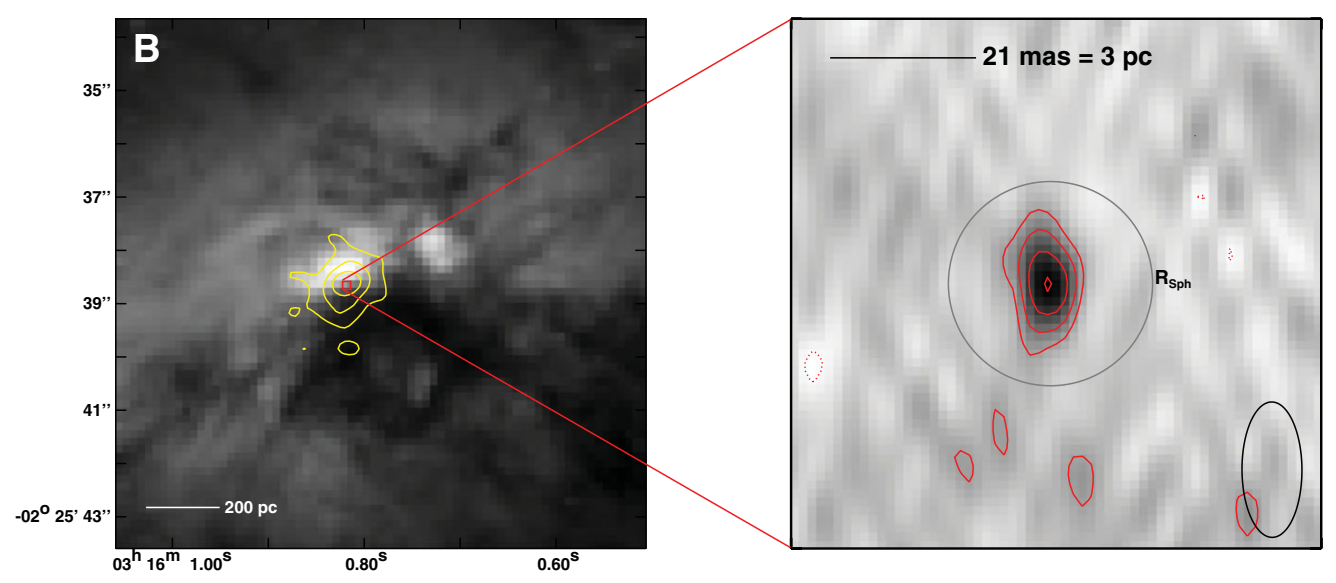

Figure 1. (Left): NGC 1266 Hubble Space Telescope $B$-band greyscale imaged. CARMA $\mathrm{CO}(1-0) v_{\text {sys }}$ contours are overlaid in yellow at levels of $[0.25,0.5,0.75]$ of the peak CO emission ( $1937 \mathrm{~K} \mathrm{~km} \mathrm{~s}^{-1}$ ). The box shows the field-of-view of the VLBA image to the right. (Right): NGC $12661.65 \mathrm{GHz}$ continuum image with contours. The VLBA beam is the black ellipse in the lower right corner and has a major axis diameter of 9.75 mas $(1.14 \mathrm{pc})$. The relative contour levels are $[-3,3,6,10,14]$ and the unit of the contour level is $42 \mu \mathrm{Jy}_{\text {beam }}{ }^{-1}$. The AGN is detected to $14 \sigma$ significance, and the VLBA does indeed probe the sphere of influence $\left(R_{\mathrm{Sph}}\right)$, but does not appear to resolve the point source.

\section{References}

Alatalo, K., et al. 2011, ApJ, 735, 88

Baldry, I. K., et al. 2004, ApJ, 600, 681

Bell, E. F., et al. 2004, ApJ, 608, 752

Cappellari, M., et al. 2011, MNRAS, 413, 813

Faber, S. M., et al. 2007, ApJ, 665, 265

Hopkins, P. F., et al. 2005, ApJ, 630, 705

Murray, N., Quataert, E., \& Thompson, T. A. 2005, ApJ, 618, 569

Schawinski, K., et al. 2007, MNRAS, 382, 1415

Strateva, I., et al. 2001, AJ, 122, 1861

Springel, V., di Matteo, T., \& Hernquist, L. 2005, MNRAS, 361, 776 